\title{
Image Retrieval Based on Gaussian Mixture Approach to Color Localization
}

\author{
Maria Luszczkiewicz-Piatek ${ }^{1}$ and Bogdan Smolka ${ }^{2}$ \\ 1 University of Lodz, Faculty of Mathematics and Computer Science, \\ Department of Applied Computer Science, Banacha 22, 90-238 Lodz, Poland \\ mluszczkiewicz@math.uni.lodz.pl \\ 2 Silesian University of Technology, Department of Automatic Control, \\ Akademicka 16 Str, 44-100 Gliwice, Poland \\ bogdan.smolka@polsl.pl
}

\begin{abstract}
The paper focuses on the possibilities of color image retrieval of the images sharing the similar location of particular color or set of colors present in the depicted scene. The main idea of the proposed solution is based on treating image as a multispectral object, where each of its spectral channels shows locations of pixels of 11 basis colors within the image. Thus, each of the analyzed images has associated signature, which is constructed on the basis of the mixture approximation of its spectral components. The ability of determining of highly similar images, in terms of one or more basic colors, reveals that the proposed method provides useful and efficient tool for robust to impulse distortions image retrieval.
\end{abstract}

Keywords: color image retrieval, color composition, Gaussian mixture.

\section{Introduction}

The rapid growth in number of images acquired and available via World Wide Web creates a need for constant improvement of existing methods of efficient management and analysis of the vast amount of data.

Therefore, analyzing this huge amount of visual information for retrieval purposes, especially when a very specific retrieval criterion is set, still remains a challenging task and therefore there are many attempts to address it [12].

One of the fields intensively explored in this area is image retrieval, providing tools and methods addressing users needs concerning finding the images which are the retrieval of images which are considered similar taking into the account the spatial location of the colors within the depicted scene.

Spatial organization of colors has been recently explored in form of spatial statistics between color pixels, such as correlograms [3] or some filter outputs [4566]. Related approaches use points of interest similarly to classic object recognition methods [8] and many other retrieval methods rely on segmentation as a basis for image indexing [9 10 1112]. Mutual arrangements of regions in images are also the basis of the retrieval, however the representation of the relationship can be non-symbolic [13].

Thus, the problem addressed in this paper is as follows. Given a color image (query), the user expects to be provided with a set of candidate images sharing the same color arrangement as the query image, i.e. the same chosen color should be present in the 
same location within the query image and the candidate images. Moreover, the user can decide whether the color similarity is analyzed in terms of the occurrence of one or more basic colors.

Although, many approaches explored the idea of the image similarity, expressed by color localization within the scene depicted by the image, the solution proposed in this paper provides a method which is robust to color outliers i.e. isolated pixels or small groups of pixels of color significantly different than that of their neighborhood. Such an approach provides a possibility of the construction of a compact structure of the image signature, which is, in case of the analyzed solution, the set of Gaussian Mixture Model (GMM) parameters, [14 15].

Therefore, the color image is represented as multispectral image formed of 11 binary images, representing the occurrence of certain base colors in particular locations. Each spectral image is then approximated by a Gaussian Mixture Model.

This approach offers the advantage of robustness to some pixel distortions, e.g. impulse noise or compression artifacts. This is obtained due to smoothing properties of Gaussian Mixture modeling. Moreover, when the model complexity is not unreasonably excessive, it can be assured that all redundant information is not modeled, i.e. is not introduced into retrieval and comparison process.

The analysis of the parameters of the evaluated models for the images for the images from the database of Wang [9] (of 1000 color images) enables to indicate the most similar pictures, sharing the same colors in the same spatial locations within the image.

The paper is organized as follows. Firstly, color categorization and the construction the spectral images are presented. Secondly, the modeling of the binary spectral images by mixtures of Gaussians is presented. Then, the evaluated results are shown and discussed. Finally, conclusions along with the remarks on future work are drawn.

\section{Color Categorization and Spectral Image Formation}

The first problem related with the color similarity among the image regions is bounded with the definition of the color similarity i.e. whether two colors are enough perceptually similar for human spectator to be considered as being the same. This problem is addressed by the approach based on the FEED[16] color categorization technique. This empirical categorization model enables to express each of HSI colors as one of 11 basic colors: red, green, blue, yellow, brown, orange, violet, pink, black, white or gray. This color space segmentation method is based on the Fast Exact Euclidean Distance (FEED) transform on the color markers placed on 2D projections of HSI color space. These color markers were a result of the empirical experiments, where human subjects were asked to categorize color stimuli into mentioned the 11 color categories.

Thus, the first step of the proposed solution is the categorization of the colors present in the given image. Therefore, each possible image is represented in terms of maximum 11 colors, as shown in Fig 1

Having the image palette decreased, the analyzed image can be seen as a multispectral object i.e. each spectral image, related to the one of the basic colors, is the binary image representing the spatial occurrence of pixels of this color. Moreover, each spectral image indicates the set of color pixels, which were recognized by the FEED model as as belonging to the same color class. 

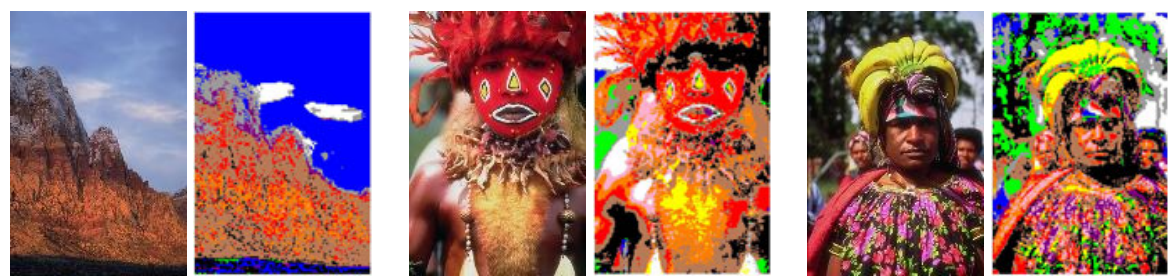

Fig. 1. Exemplary results (right) of the original color image (left) categorization based on FEED technique for 11 base colors evaluated for 3 images of the database of Wang [9]

\section{Mixture Modeling of Spectral Data}

The next step of the proposed technique is the modeling of each of the spectral images using the Gaussian Mixture Model [14|15] and Expectation-Maximization algorithm (EM), [17].

In order to apply the proposed technique, each of binary spectral images are treated as histogram $\Phi(x, y)$, where $x$ and $y$ denotes the image size. This histogram is subjected then to mixture modeling. This approach describes color regions (in specified channel), present in depicted scene, using combination of 'blobs' (Gaussians).

However, to apply the proposed technique, and thus to assure the comparability of the evaluated results, the multispectral images, representing images stored in the analyzed database, should be resized using a method which does not introduce any new colors into a depicted scene during the resizing process.

For the need of this work the used Gaussian Mixture Model is obtained running 75 times the EM procedure for 7 model components. The choice of the model complexity and number of iterations is in general a trade-off between accuracy of the evaluated model and the obtained visual similarity, when some data should be excluded from further consideration. However, in the methodology proposed in this work, the main reasoning for such a choice of modeling parameters is the fact that the exact modeling of spectral data is not desirable, because of the fact that there can be data which should not be incorporated in further analysis, e.g. noise or small insignificant homogenous color regions, which do not convey any useful information. Thus, any possibly irrelevant data will be not considered, [18 19 20 21].

At this stage of the algorithm each image can be seen as set of spectral images, which are modeled using Gaussian Mixture Model, forming it signature, as shown in Fig 2.

The evaluation of the GMM of the spectral image is illustrated by Fig. 3 . Each spectral image, which is binary image conveying information about the spatial location of certain color, is approximated by GMM, reflecting the main structure of the spectral image content.

\section{Image Retrieval}

Having the spectral images modeled, the user can decide which color or colors reflects the similarity among the images. Therefore, the set of colors which will be taken into 

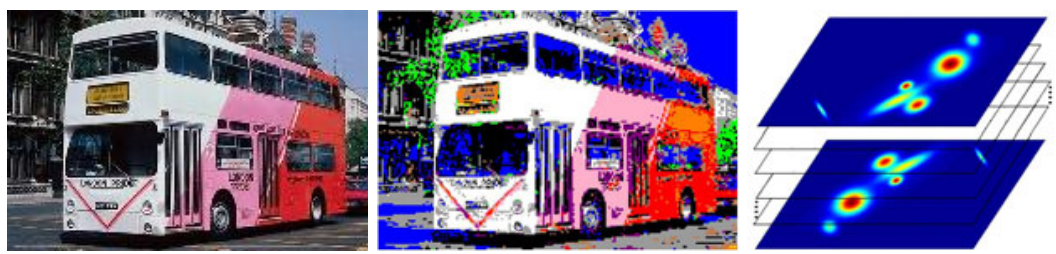

Fig. 2. The construction of the set of GMM models which is a basis for the proposed image retrieval. The original image (left) was subjected to color categorization technique based on FEED model (middle) and each of the spectral images corresponding to 11 basic colors are modeled using GMM approach (right).
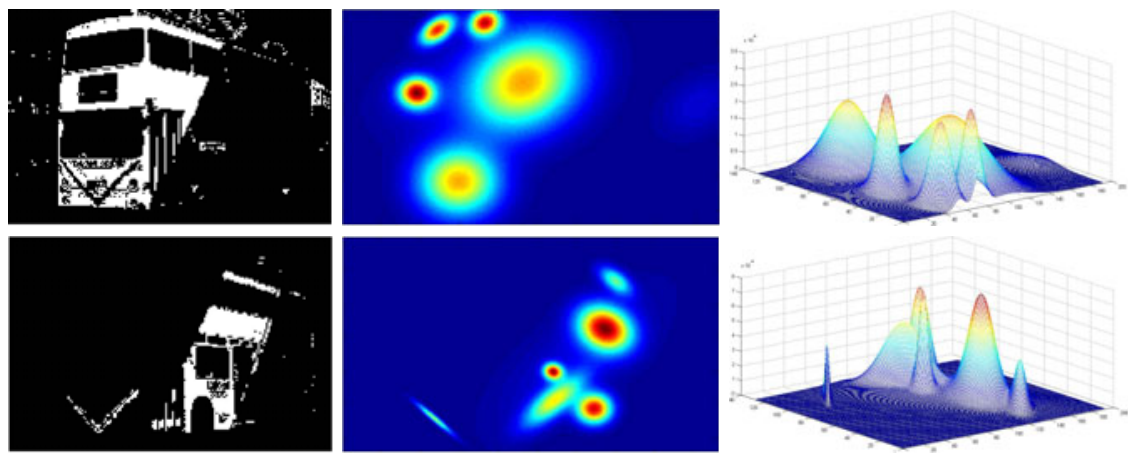

Fig. 3. The evaluation of the mixture model of spectral image corresponding to a chosen color (left) for original image shown in Fig. 2 The 2D (middle) and 3D (right) visualization of GMM are the basis for the retrieval.

account should be chosen. The mixture models of spectral images, corresponding to the user choice, are compared to GMM of respective spectral images of a given query.

For the user choice of colors, the index $\eta$, representing each database image, is constructed in relation to query image. The evaluated index also depends on the number of chosen colors, according to:

$$
\eta_{i}=\sum_{k=1}^{m} d\left(Q_{k}, I_{i, k}\right)
$$

where $m$ denotes the number of spectral images (colors) taken into account during retrieval process, $Q$ and $I$ represent query and analyzed image from the database, indexed by $i$ respectively. The distance between the spectral images $Q_{k}$ and $I_{i, k}$ can be defined as Minkowski distance (e.g. $L_{1}$ ) or Earth Mover's Distance (EMD), [11]. The EMD is based on the assumption that one of the histograms reflects "hills" and the second represents "holes" in the ground of a histogram. The measured distance is defined as a minimum amount of work needed to transform one histogram into the other using a "soil" of the first histogram. As this method operates on signatures and their weights using GMM, we assigned as signature values the mean of each component and for the signature weight the weighting coefficient of each Gaussian in the model. 
In details, let us assume that a user is interested in retrieval of images similar to a given query, having regions of the perceptually similar colors in corresponding locations. All images are subjected to the color categorization, mixture modeling, and similarity-to-query index construction. The exact value of the image index is bounded to the number of chosen colors, in terms of which, images are being compared. For each pair of the corresponding spectral images (of the query and the analyzed images) their mutual similarity is evaluated. Knowing that each spectral image is represented by a mixture model, the similarity evaluation resolves to the calculation of the similarity between evaluated GMM's for a corresponding color. The similarity indices for each spectral level are then combined to create the overall similarity-to-query index $\eta$ for each image in the analyzed database. On the basis of those indexes the candidate images the most similar to given query are selected.

Let us note that when only a small set of basis color are chosen, the retrieved images will be similar only in terms of those colors, that can lead to the perceptual sensation that candidate images are in fact dissimilar when the overall image composition is taken into account. Moreover, not only the the undesirable data (as noise) can be omitted in the modeling process, but also the relatively small regions, in comparison to overall image size, can be not included into the mixture model. The ratio of data which is modeled to overall image data depends on the complexity of the used mixture model. Thus, increasing the model complexity, on the one hand, leads to more accurate reflection of the given data, but on the other hand, there is a possibility that redundant data will be taken into consideration.

\section{Retrieval Results}

The presented experiments were evaluated in two steps. Firstly, the color similarity was tested for a single color, i.e. images were retrieved only on the basis of the similarity of one chosen spectral image corresponding to a single chosen color (Fig. 4). The second part of the experiment was concentrated on the retrieval based on the combination of colors (see Figs. 5] and 6).

Let us note, that when the retrieval process is based only on a single color there is a chance that the overall similarity sensation can be different that those suggested by single color comparisons. However, these experiments prove the efficiency of the proposed methodology.

Moreover, the evaluated results for single color comparison for $L_{1}$ and $E M D$ measures produce still accurate but slightly different sets of candidate images, because the first of the similarity measures does not take into account any, even slight spatial shift, of the location of the color regions in spectral image. The second of the similarity measures $E M D$, due to its inherent properties, allows small changes in color spatial location to claim similarity. However, this phenomenon does not occurs when multiple colors are analyzed simultaneously (as illustrated by Fig. 5 and 6), because when the numerous criteria are applied, only a smaller subset of images are capable to satisfy those conditions. In case of analyzed databases, chosen for the experiments described in this paper, 


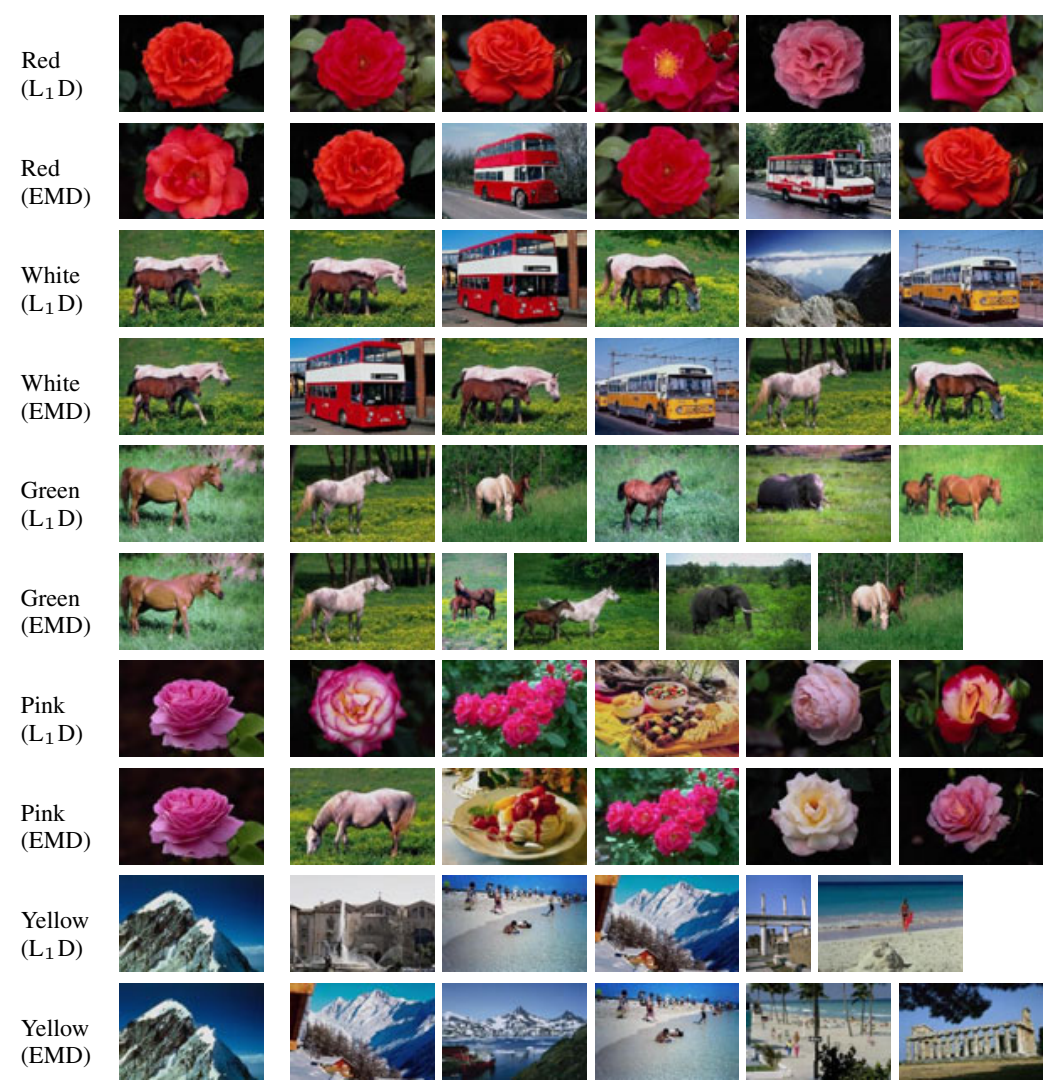

Fig. 4. The results of the retrieval evaluated on the basis of the mixture approximation of spectral images corresponding to the single color chosen by user, evaluated for the database of Wang [9]. For the similarity measure for each color the $L_{1}$ or $E M D$ methods we used.

the possible differences in evaluated results are unnoticeable. When the database of significantly larger size would be taken into account, these differences would be visible.

There is open question which of the similarity measure is more accurate. To address this problem, also the other aspects should be considered, such as the fact that for the $E M D$ similarity measure the evaluation of the image signature, which need to be stored for further analysis, is reduced to set of GMM parameters. In case of pixel by pixel comparisons (as for $L_{1}$ measure), the completely evaluated model of 11 mixtures should be stored, which not always is acceptable. 


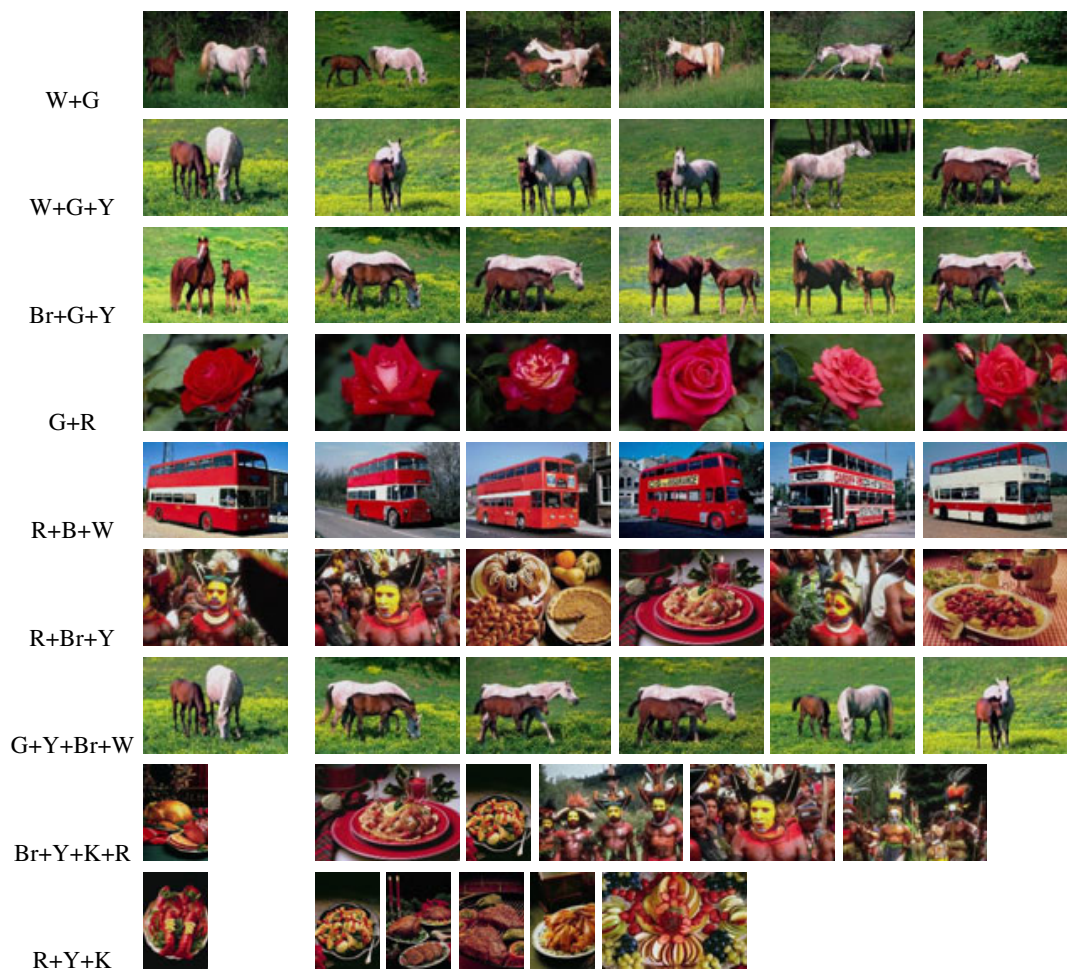

Fig. 5. The results of the retrieval evaluated on the basis of the mixture approximations of a set spectral images corresponding to the colors chosen by user. The experiments tested the efficiency of proposed method for the combination of the following colors, where: white, green, yellow, brown, red, blue and black are denoted by letters W, G, Y, Br, R, B and K.

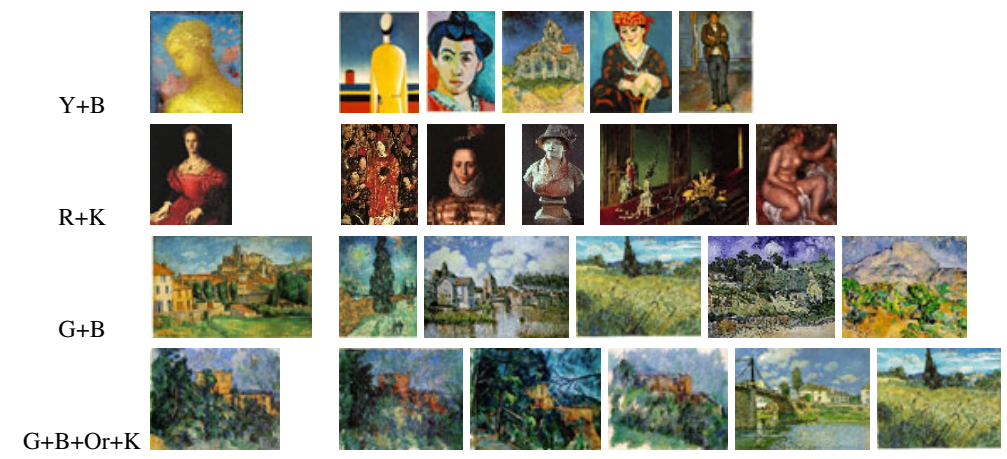

Fig. 6. The results of the retrieval evaluated on the basis of the proposed method for the images of database of Webmuseum (http://www.ibiblio.org/wm/). The experiments tested the efficiency of proposed method for the combination of the following colors, where: yellow, blue, red, black, green and orange are denoted by letters $\mathrm{Y}, \mathrm{B}, \mathrm{R}, \mathrm{K}, \mathrm{G}$ and $\mathrm{Z}$. The results evaluated for $L_{1}$ and $E M D$ similarity measures provide the equivalent results. 


\section{Conclusions and Future Work}

In this paper we present a novel method operating on color images treated as multispectral images, combined of the set of the binary images representing each of 11 basic colors. The experiments evaluated on images of database of Wang tested the effectiveness of the proposed method when only one color was taken as the similarity criterion, as long as when image similarity was based on the set of colors.

As illustrated by Figs. 4, 5 and 6 the technique described in this paper, utilizing the Gaussian mixture modeling approach to spatial location of color pixel within the color image, offers satisfactory retrieval results. Moreover, proposed technique is robust, due to the approximating nature of GMM, to any impulse noise or redundant information.

Future work will be based on the exploration of the robustness of the proposed technique to possible image deteriorations, along with the comparison of the effectiveness of the retrieval process in comparison to other existing methods, taking into account the spatial location of the analyzed colors. Moreover, these experiment will be evaluated on more numerous databases that that of Wang.

\section{References}

1. Datta, R., Joshi, D., Li, J., Wang, J.Z.: Image Retrieval: Ideas, Influences, and Trends of the New Age. ACM Computing Surveys 40(2), 1-60 (2008)

2. Zhou, X.S., Rui, Y., Huang, T.S.: Exploration of Visual Data. Kluwer, Dordrecht (2003)

3. Huang, J., et al.: Spatial Color Indexing and Applications. International Journal of Computer Vision 35(3), 245-268 (1999)

4. Pass, G., Zabih, R.: Comparing images using joint histograms. Journal of Multimedia Systems 7(3), 234-240 (1999)

5. Ciocca, G., Schettini, L., Cinque, L.: Image Indexing and Retrieval Using Spatial Chromatic Histograms and Signatures. In: Proc. of CGIV, pp. 255-258 (2002)

6. Lambert, P., Harvey, N., Grecu, H.: Image Retrieval Using Spatial Chromatic Histograms. In: Proc. of CGIV, pp. 343-347 (2004)

7. Hartut, T., Gousseau, Y., Schmitt, F.: Adaptive Image Retrieval Based on the Spatial Organization of Colors. Computer Vision and Image Understanding 112, 101-113 (2008)

8. Heidemann, G.: Combining Spatial and Colour Information For Content Based Image Retrieval. Computer Vision and Image Understanding 94, 234-270 (2004)

9. Wang, J.Z., Li, J., Wiederhold, G.: SIMPLIcity: Semantics-Sensitive Integrated Matching for Picture Libraries. IEEE Trans. Patt. Anal. Mach. Intel. 9, 947-963 (2001)

10. Rugna, J.D., Konik, H.: Color Coarse Segmentation and Regions Selection for Similar Images Retrieval. In: Proc. of CGIV, pp. 241-244 (2002)

11. Dvir, G., Greenspan, H., Rubner, Y.: Context-Based Image Modelling. In: Proc. of ICPR, pp. 162-165 (2002)

12. Jing, F., Li, M., Zhang, H.J.: An Effective Region-Based Image Retrieval Framework. IEEE Trans. on Image Processing 13(5), 699-709 (2004)

13. Berretti, A., Del Bimbo, E.: Weighted Walktroughs Between Extended Entities for Retrieval by Spatial Arrangement. IEEE Trans. on Multimedia 3(1), 52-70 (2002)

14. McLachlan, G., Peel, D.: Finite Mixtures Models. John Wiley \& Sons, Chichester (2000)

15. Bilmes, J.: A Gentle Tutorial on the EM Algorithm and its Application to Parameter Estimation for Gaussian Mixture and Hidden Markov Models. University of Berkeley, ICSI-TR-97021 (1997) 
16. Van den Broek, E.L., Schouten, T.E., Kisters, P.M.F.: Modeling human color categorization. Pattern Recogn. Lett. 29(8), 1136-1144 (2008)

17. Dempster, A., Laird, N., Rubin, D.: Maximum Likelihood from incomplete data. J. Royal Stat. Soc. 39B, 1-38 (1977)

18. Luszczkiewicz, M., Smolka, B.: Gaussian Mixture Model Based Retrieval Technique for Lossy Compressed Color Images. In: Kamel, M.S., Campilho, A. (eds.) ICIAR 2007. LNCS, vol. 4633, pp. 662-673. Springer, Heidelberg (2007)

19. Luszczkiewicz, M., Smolka, B.: A Robust Indexing and Retrieval Method for Lossy Compressed Color Images. In: Proc. of IEEE International Symposium on Image and Signal, Processing and Analysis, pp. 304-309 (2007)

20. Luszczkiewicz, M., Smolka, B.: Spatial Color Distribution Based Indexing and Retrieval Scheme. In: Advances in Soft Computing, vol. 59, pp. 419-427 (2009)

21. Luszczkiewicz, M., Smolka, B.: Application of Bilateral Filtering and Gaussian Mixture Modeling for the Retrieval of Paintings. In: Proc. of PODKA, vol. 3, pp. 77-80 (2009)

22. Rubner, Y., Tomasi, C., Guibas, L.J.: The Earth Mover's Distance as a Metric for Image Retrieval. International Journal of Computer Vision 40(2), 99-121 (2000) 